\title{
Influence of ion implantation on the near-surface structure of thin Ni and Pd films on lithium niobate and lithium tantalate
}

\author{
V.O. Lysiuk ${ }^{1,2}$, V.S. Staschuk ${ }^{1}$, M.I. Kluy ${ }^{2}$, O.V. Vakulenko ${ }^{1}$, L.V. Poperenko ${ }^{1}$ \\ ${ }^{1}$ Department of Physics, Taras Shevchenko Kyiv National University \\ 1, Bld., 2, Academician Glushkov prospect, 03022 Kyiv, Ukraine \\ Phone: +380 (44) 526-22-96; fax: +380(44) 526-45-07 \\ ${ }^{2} V$. Lashkaryov Institute of Semiconductor Physics, NAS of Ukraine, \\ 41, prospect Nauky, 03028 Kyiv, Ukraine; e-mail: lysiuk@univ.kiev.ua
}

\begin{abstract}
The systems "thin Ni film - lithium niobate" and "thin Pd film - lithium tantalate” are implanted by $\mathrm{Ar}^{+}$ions with an energy of $100 \mathrm{keV}$ and a dose of $10^{16} \mathrm{~cm}^{-2}$. Analyses of the systems by AFM and SEM have shown that the ion implantation essentially modifies the near-surface structure resulting in a change of its optical, electrical, and mechanical properties. Strong difference in the near-surface structures between implanted systems with $\mathrm{Ni}$ or Pd thin films is observed. Such a difference is explained by the heterogeneity of an ion beam and different properties of the materials. The application to the development of high-sensitive pyroelectric detectors with high damage threshold is proposed.
\end{abstract}

Keywords: ion implantation, thin films, pyroelectric detector.

Manuscript received 04.04.07; accepted for publication 24.04.07; published online 19.10.07.

\section{Introduction}

Lithium niobate and lithium tantalate are widely used nonlinear crystals with unique electro-optical, acoustic, piezoelectric, pyroelectric, and nonlinear optical properties making them suitable materials for applications in different optoelectronic devices, particularly in pyroelectric detectors [1-3]. Owing to the high Curie temperature, good chemical and mechanical stability, and high optical damage threshold, $\mathrm{LiNbO}_{3}$ and $\mathrm{LiTaO}_{3}$ are the best materials for high-power precision measurements that require long-time stability and durability. The TGS and DTGS are the most sensitive pyroelectric materials owing to the highest value of their pyroelectric coefficients [4-5]. But the sensitivity to humidity and the low damage threshold make the mentioned materials unsuitable for high-power measurements and space applications.

The problem was solved by sputtering a thin absorbing layer on such pyroelectrics as lithium niobate and lithium tantalate in order to increase the absorption, i.e. sensitivity [6-7]. Golden black is a very good and widely used material for absorbing layers of different types of photodetectors. Owing to its colloidal structure, golden black makes the spectral sensitivity nonselective in the visible and infrared ranges. But its adhesion to a pyroelectric material is too low that results in a decrease of the damage threshold [8]. Thin metal films absorb light also well, but the problem is the same: low adhesion between absorbing film and substrate.

The well-known, but insufficiently studied method for a modification of the surface and near-surface structure is ion implantation [9-11]. Such effects as implantation of recoil atoms, radiation-accelerated diffusion, atom intermixing, etc. accompany usually the process of ion implantation. With the purpose to increase the adhesion between a thin $\mathrm{Ni}(\mathrm{Pd})$ film and lithium niobate (lithium tantalate), we will study the influence of ion implantation on the near-surface structure and optical properties of the systems "thin Ni film - lithium niobate" and "thin Pd film - lithium tantalate”.

\section{Experiment}

The slices of lithium niobate and lithium tantalate with thicknesses of $100 \mu \mathrm{m}$ were cut from the corresponding single crystals with orientation of polarizability in parallel with the surface area of samples. Thin Ni and Pd films with thicknesses of 15, 20, 30, and $40 \mathrm{~nm}$ were deposited on this substrate by the ion-plasma method in a magnetron. Ion implantation of the systems "thin $\mathrm{Ni}$ film - lithium niobate" and "thin Pd film - lithium tantalate" was carried out by $\mathrm{Ar}^{+}$ions with an energy of $100 \mathrm{keV}$ and a dose of $10^{16} \mathrm{~cm}^{-2}$ using a "Vezuvii 2/450" ion implanter. The energy of ions and the dose were calculated by the Monte-Carlo method under 
condition that a peak of the distribution of ions lies at the interface "thin metallic film - pyroelectric".

It is necessary to note that sharp changes in the structure of the samples with deterioration of their properties were observed at ion energies $E>400 \mathrm{keV}$. At sufficiently small ion energies $E<25 \mathrm{keV}$, the implantation practically doesn't essentially influence the surface structure of the samples. The optimal doses of $\mathrm{Ar}^{+}$ions are from $10^{15}$ to $10^{17} \mathrm{~cm}^{-2}$. Sufficiently larger doses cause changes in the structure and properties of the samples and the deterioration of their functional characteristics.

It was necessary to reach a maximum adhesion of the $\mathrm{Ni}$ and $\mathrm{Pd}$ films to the substrate without both essential changes in the structure and the deterioration of the properties of the absorbing film and the lithium niobate (lithium tantalate) substrate as well.

For this purpose, annealing and a further treatment of the samples were carried out in order to decrease inhomogeneities in the systems. Such inhomogeneities appear on the deposited metallic films on the substrate during the ion implantation. Minimization of the most part of defects of the lithium niobate (lithium tantalate) substrate is a necessary operation to improve the characteristics of the samples.

Investigations of the surface structure were made by a Scanning Electron Microscope DTGS-35 and a Field Emission Scanning Electron Microscope ULTRA 55 as well as Atomic Force Microscopy. The nearsurface structure of the systems "thin Ni film - lithium niobate" and "thin Pd film - lithium tantalate" were investigated before and after the implantation by $\mathrm{Ar}^{+}$ ions. Qualitative and quantitative chemical analyses of the systems were controlled by the INCA Energy SEM system of microanalysis with support of an INCA Xsight detector and an INCA X-stream module.

\section{Results and discussion}

The SEM investigation of the surface structure of nonimplanted systems "thin Ni film - lithium niobate" and "thin Pd film - lithium tantalate" has shown that the surface structures of both samples are smooth [12]. Some defects that were observed on the surface of the samples (Fig. 1a) are related to heterogeneity of the metal film deposition and some mechanical microdamages. At a larger magnification (Fig. 1b), the granular structure characteristic of metals was observed with the average dimension of clusters of about $50 \mathrm{~nm}$. Of most interest are the SEM and AFM investigations of the implanted systems.

The SEM investigations of the implanted systems "thin Ni film - lithium niobate" and "thin Pd film lithium tantalate" have shown (Fig. 2) that the surface structure is changed after the ion implantation. According to Fig. 2a, the surface structure of the systems "thin $\mathrm{Ni}$ film - lithium niobate" become coated by bubbles after the implantation. The dimensions of bubbles vary from $100 \mathrm{~nm}$ to $2 \mu \mathrm{m}$. The appearance of bubbles is explained

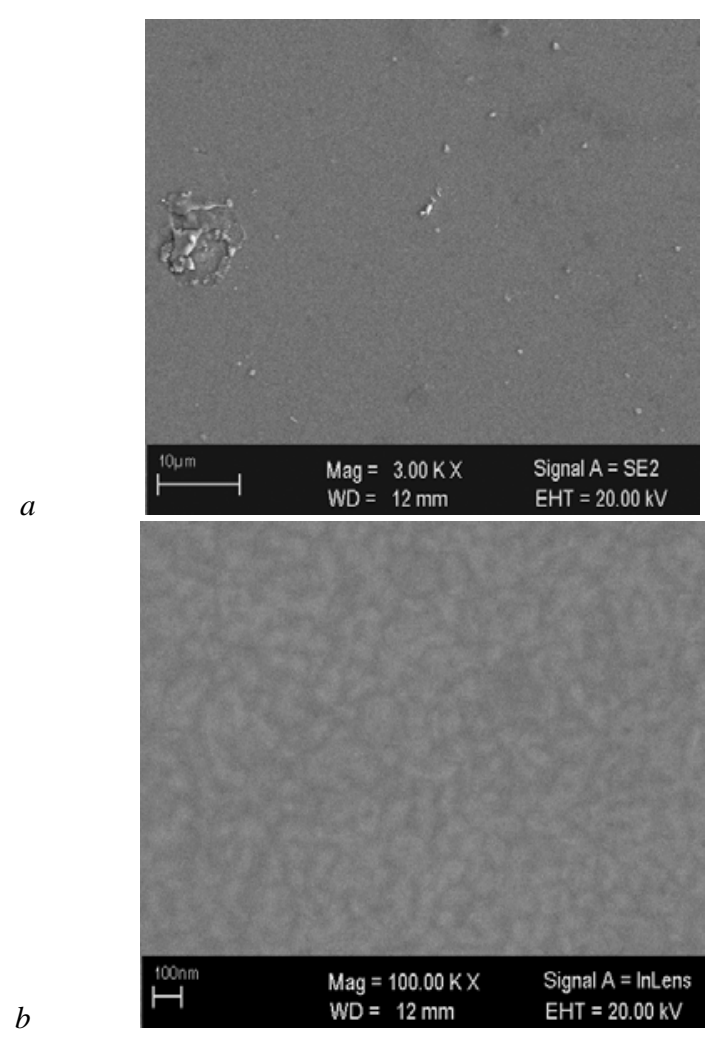

Fig. 1. SEM images of nonimplanted systems "thin Ni film lithium niobate" with a $\mathrm{Ni}$ film thickness of $30 \mathrm{~nm}$ and a substrate one of $100 \mu \mathrm{m}$. Magnification: $3 \times 10^{3}$ (a) and $10^{5}$ (b).

by the sharp rise of a temperature at the areas, where the maximal local densities of implanted ions are located. As a result, the sharp microboiling with birth of bubbles takes place during the ion implantation. Owing to the high heat conductivity of $\mathrm{Ni}$, a further heat exchange with the rest material of the system and the environment causes the congelation of heated areas. This selective effect exists due to fluctuations of the density of implanted ions under consideration in different surface areas with very small dimensions (for example, $1 \mu^{2}$ ). The integral of the density of local ions gives a total dose of ions of $10^{16} \mathrm{~cm}^{-2}$.

The analysis of the system "thin Pd film - lithium tantalate" implanted by $\mathrm{Ar}^{+}$ions has shown (Fig. 2a) that the deep etched areas of different forms are observed on the surface of a sample after the implantation. The dimensions of the observed structures vary from 2 to $100 \mu \mathrm{m}$. The appearance of etched areas testifies that mechanical properties of the system "thin Pd film lithium tantalate" strongly differ from those of the system "thin Ni film - lithium niobate". Ion bombardment causes a peeling, i.e. a damage of surface areas with maximal local dose of implanted ions. Fig. 2b represents the magnified SEM image of the same system that shows the detailed structures of etched areas. The depth of the structures exceeds the Pd film thickness equal to $30 \mathrm{~nm}$, so the ion implantation destroys not only 


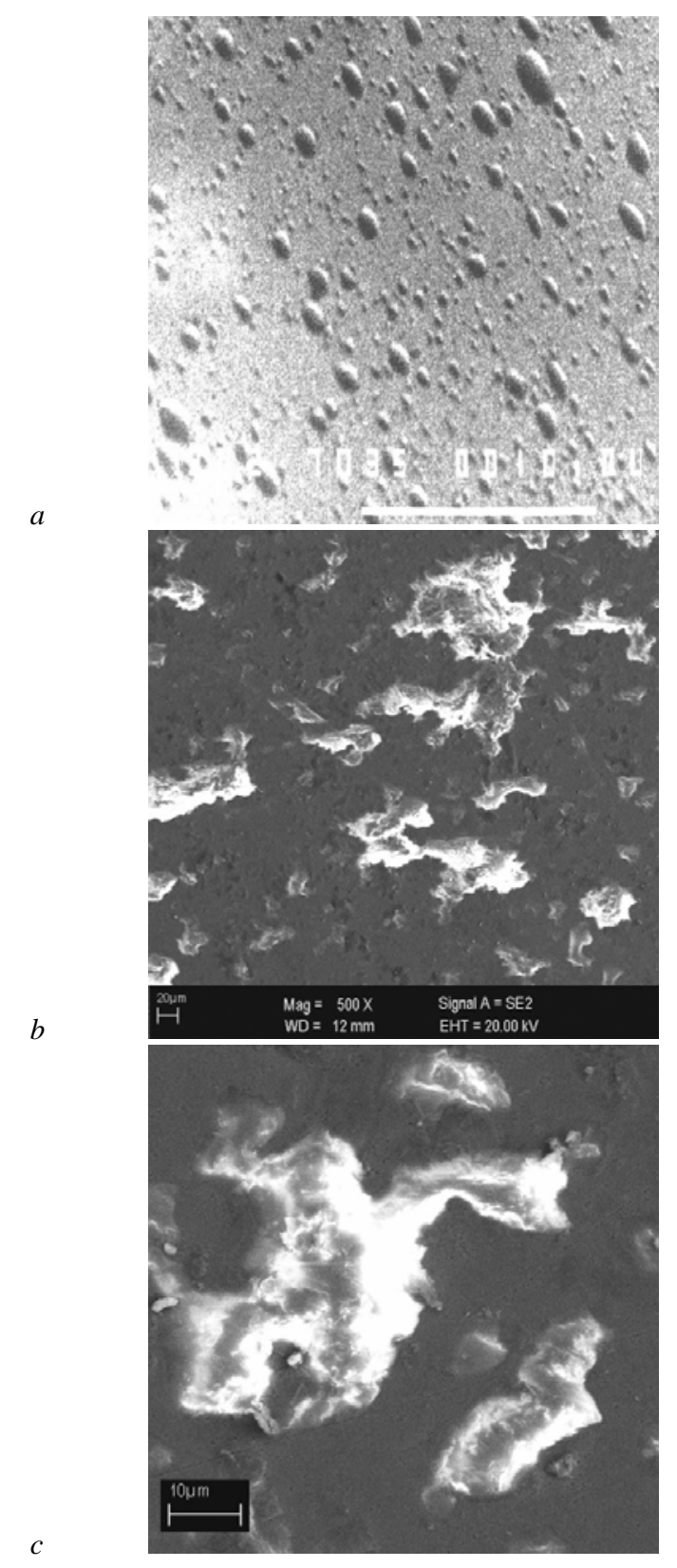

Fig. 2. SEM image of implanted system "thin Ni film lithium niobate" made by SEM DTGS-35 (a), and SEM images of the implanted system "thin Pd film - lithium tantalate” made by FESEM ULTRA 55 with low (b) and high (c) magnification.

the Pd film, but also the subsurface layer of lithium tantalate, which was not observed for a thin Ni film on lithium niobate.

Investigations of near-surface structures of the ionimplanted systems "thin Ni film - lithium niobate" and "thin Pd film - lithium tantalate” by AFM (Fig. 3) have confirmed the results obtained by SEM. Fig. 3a demonstrates some magnified bubbles on the surface of a Ni film. At the center of the image, the bubble with a diameter of about $250 \mathrm{~nm}$ is located near one of the
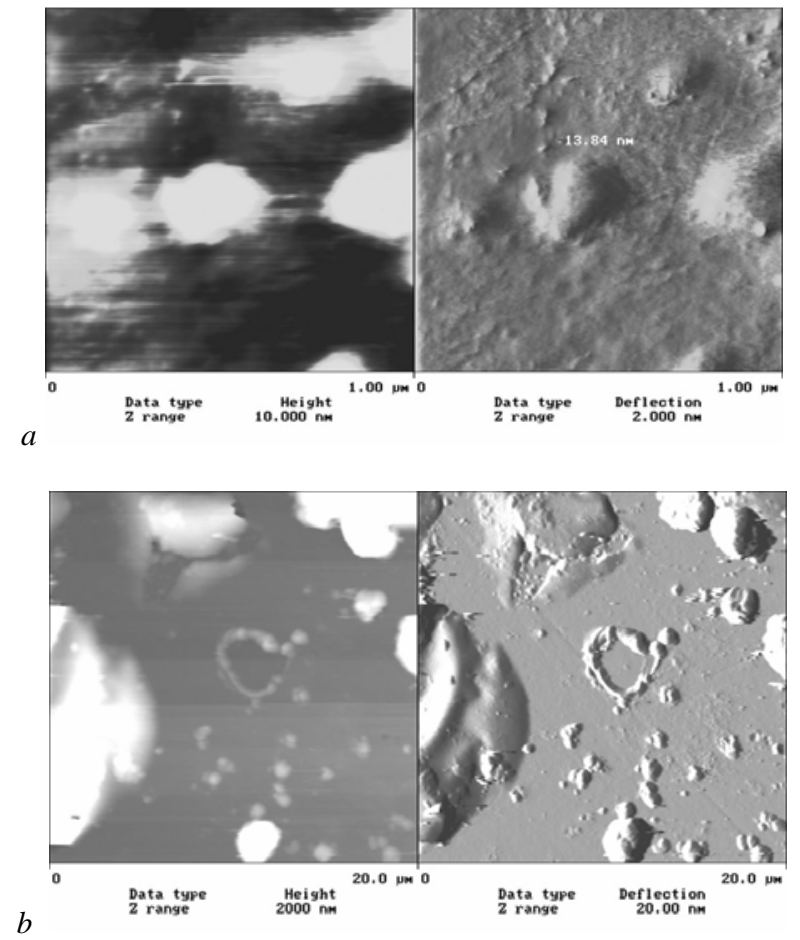

Fig. 3. AFM images of ion-implanted systems "thin Ni film lithium niobate" (a) and "thin Pd film - lithium tantalate" (b).

smallest bubbles with a diameter of about $14 \mathrm{~nm}$. The dimension of bubbles is proportional to the total local dose of implanted ions and the local flow of ions $F=N /(t \cdot S)$, where $F$ - local flow of ions, $N$ - number of ions, $t$ - time, and $S$ - area $\left(S \sim 1 \ldots 100 \mu \mathrm{m}^{2}\right)$.

The AFM image of a thin Pd film on lithium tantalate has shown that, during the ion implantation, heated particles of the Pd film are dispersed from the areas of maximal concentration of ions to the film surface. Deep etched areas together with particles sputtered from their regions make the surface of the system enough rough, which results in a rise of the absorption of such a system in the visible and infrared ranges as well.

The qualitative and quantitative (Table) analyses of the implanted systems "thin Ni film - lithium niobate" and "thin Pd film - lithium tantalate" have practically confirmed the chemical composition of the samples. The absence of $\mathrm{Li}$ is explained by the impossibility to register such a low-weight metal by the X-ray method using the INCA Energy SEM system of microanalysis. The presence of carbon additionally to $\mathrm{Ni}, \mathrm{Nb}$, and $\mathrm{O}$ in the ion-implanted system "thin $\mathrm{Ni}$ film - lithium niobate" testifies that, during the metal film deposition, the ion implantation, or X-ray investigation, this chemical element was dispersed from other samples or from the inner part of the devices. Analyzing the chemical composition of the system "thin Pd film -

(C) 2007, V. Lashkaryov Institute of Semiconductor Physics, National Academy of Sciences of Ukraine 
Table. Quantitative analysis of chemical elements in the implanted systems "thin Ni film - lithium niobate" and "thin Pd film - lithium tantalate".

\begin{tabular}{|l|l|l|l|l|l|}
\hline \multicolumn{3}{|l|}{$\begin{array}{l}\text { Thin Ni film - lithium } \\
\text { niobate }\end{array}$} & \multicolumn{3}{l|}{$\begin{array}{l}\text { Thin Pd film - lithium } \\
\text { tantalate }\end{array}$} \\
\hline Element & $\begin{array}{l}\text { Weight } \\
\%\end{array}$ & $\begin{array}{l}\text { At. } \\
\%\end{array}$ & Element & $\begin{array}{l}\text { Weight } \\
\%\end{array}$ & At. \% \\
\hline C K & 4.56 & 19.58 & C K & 11.81 & 42.79 \\
\hline O K & 6.2 & 4.51 & O K & 14.23 & 38.72 \\
\hline Ni K & 81.44 & 71.58 & Pd L & 4.07 & 1.67 \\
\hline Nb L & 7.79 & 4.33 & Ta M & 69.89 & 16.82 \\
\hline Total & 100.00 & & Total & 100.00 & \\
\hline
\end{tabular}

lithium tantalate," we have found carbon additionally to the expected Ta, $\mathrm{O}$, and Pd. The presence of $\mathrm{C}$ is often observed during $\mathrm{X}$-ray investigations in different samples where it is not expected to be measured.

The mentioned changes in the near-surface structure are accompanied by a strong increase of the adhesion between the metal film and the pyroelectric substrate for both systems owing to the atom intermixing during the ion implantation. This is a very successful result in the case of the use of the investigated systems in pyroelectric detectors. Such an increase of the adhesion causes a rise of the damage threshold of pyroelectric detectors fabricated on the base of implanted systems. This makes this system applicable to the measurements of high-power laser radiation, where batch pyroelectric detectors coated by golden black cannot be used due to a damage. Measurements have shown that the damage thresholds of both systems increase at least 100 times, which allows one to use these systems for the registration of power laser radiation.

The modification of the near-surface structure of thin Pd films on lithium tantalate by ion implantation increases their sensitivity as a result of the absorption rise. But additionally, different dimensions of etched areas make this system nonselective in the infrared range, which is of importance in spectral measurements.

\section{Conclusions}

The SEM and AFM investigations of the systems "thin Ni film - lithium niobate" and "thin Pd film - lithium tantalate" have shown that, after the implantation by $\mathrm{Ar}^{+}$ ions, the near-surface structure of the systems strongly changes in contrast to that of nonimplanted systems, where the surface structure was smooth.

A thin Ni film on lithium niobate is coated by bubbles during the ion implantation. The mechanism of the formation of bubbles consists in the heating of local areas of the film by the heterogeneous flow of ions. The local areas, into which the maximal local dose of implanted ions have penetrated, are melting. The bubbles appear as a result of the microboiling and the further congelation of bubbles swollen to different dimensions due to the heat exchange with the environment.

The system "thin Pd film - lithium tantalate" is coated by cavities with different dimensions during the implantation. The heterogeneous flow of ions causes the etching of local areas on the surface at places, where the highest local dose of implanted ions is realized. The surface structure of the system modified by ion implantation has nonselective spectral response owing to a high roughness and a variety of dimensions of cavities.

Ion implantation increases the adhesion of the thin metal film to the substrate for both systems "thin Ni film - lithium niobate" and "thin Pd film - lithium tantalate". This allows one to use them as the sensitive elements of pyroelectric detectors with high sensitivity and high damage threshold for the registration of power laser radiation. The system with a $\mathrm{Ni}$ thin film is suitable to be used for the laser radiation measurements owing to the highest damage threshold. The system "thin Pd film lithium tantalate" is excellent to be used for spectral measurements in a wide spectral range $(0.4-15 \mu \mathrm{m})$ owing to its nonselective response in the infrared range

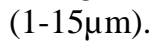

\section{References}

1. H. Chaib, T. Otto, and L.M. Eng, Electrical and optical properties of $\mathrm{LiNbO}_{3}$ single crystals at room temperature // Phys. Rev. B 67, 174109 (2003).

2. V. Caciuk, $A b$ initio zone-center phonons in $\mathrm{LiTaO}_{3}$ : Comparison to $\mathrm{LiNbO}_{3} / /$ Phys. Rev. B 64, 224303 (2001).

3. V.O. Lysiuk, L.V. Poperenko, V.S. Staschuk, and M.I. Kluy, Study of the systems thin metal film thick lithium niobate substrate implanted by $\mathrm{Ar}^{+}$ ions and its application for production of pyroelectric photodetectors // in Smart Sensors, Actuators and MEMS, Jung-Chih Chiao, V.K. Varadan, and C. Cane (Eds.), Proc. SPIE 5116, p. 818-827 (2003).

4. C.K. Sheen, T.C. Chow, M.L. Hu, Y.D. Juang, W.S. Tse, and S.J. Lin, Low temperature phase transition of triglycine sulfate studied by Raman scattering // Chinese J. Phys. 35, No. 6-11, p. 929934 (1997).

5. J. Novotny, B. Brezina, and J. Zelinka, Growth and characterization of TGS and DTGS single crystals doped with $\mathrm{Pt}(\mathrm{II}), \mathrm{Pt}(\mathrm{IV})$ and L-alanine // Cryst. Res. Technol. 39, No. 12, p. 1089-1098 (2004).

6. O.V. Vakulenko, O.V. Elfimov, L.S. Kremenchugskiy et al. // Zhurnal prikladnoy spektroskopii 53, No. 1, 159-161 (1990) (in Russian).

7. L.M.P. Pinheiro et al., Structure, morphology and composition of thin Pd and Ni films deposited by dc magnetron sputtering on polycrystalline $\mathrm{Ni}$ and Pd foils // J. Phys. D: Appl. Phys. 38, p. 4241-4244 (2005). 
8. J. Lehman, E. Theocharous, G. Eppeldauer, and C. Pannell, Gold-black coatings for freestanding pyroelectric detectors // Meas. Sci. Technol. 14, 916-922 (2003).

9. S.G. Mayr and R.S. Averback, Effect of ion bombardment on stress in thin metal films // Phys. Rev. B 68, 214105 (2003).

10. V.O. Lysyuk, L.V. Poperenko, V.S. Staschuk, Investigations of characteristics of metal film based pyroelectric detectors implanted by $\mathrm{Ar}^{+}$ ions // Semiconductor Physics, Quantum Electronics and Optoelectronics 5, No. 4, p. 412416 (2002).
11. Ion Implantation and Beam Processing, J.S. Williams and J.M. Poate (Eds.). Academic Press, New York, 1984.

12. L.V. Poperenko, M.I. Kluy, V.S. Staschuk and V.O. Lysyuk, Metal film based pyroelectric detectors and their optical characteristics // Spectroscopy of Molecules and Crystals, G.A. Puchkovska and S.A. Kostyukevych (Eds.), Proc. SPIE 4938, p. 81-84 (2002).

13. I.J. Youngs, N. Bowler, and O. Ogurlu, Dielectric relaxation in composites containing electrically isolated particles with thin semicontinuous metal coatings // $\mathrm{J}$. Phys. D: Appl. Phys. 39, p. 1312-1325 (2006). 